\title{
Terapia Nutricional na Doença Celíaca: Fatores Associados a Adesão de Crianças e Adolescentes
}

\author{
Nutritional Therapy in Celiac Disease: Factors Associated with Adherence of Children and \\ Adolescents
}

Terapia nutricional en la enfermedad celiaca: Factores asociados a la adhesión de niños y adolescentes

Maria de Fátima Sousa Barros Vilarinho ${ }^{1 *}$, Tamires da Cunha Soares ${ }^{1}$, Gilvânia da Conceição Rocha $^{2}$, Ticianne da Cunha Soares ${ }^{1}$, Mísia Joyner de Sousa Dias Monteiro ${ }^{1}$, Vanessa Moreira de Lima', Gabriel Barbosa Câmara ${ }^{3}$, Lívia de Araújo Rocha ${ }^{1}$, Mickael de Paiva Sousa ${ }^{1}$, Thamires de Carvalho Mendes ${ }^{1}$, Antonia Charliene da Silva Pereira ${ }^{1}$, Liriane Andressa Alves da Silva ${ }^{1}$, Charles Emanuel de Oliveira Silva1, Victor Brito Dantas Martins ${ }^{1}$, Victor Alves de Oliveira'

\section{RESUMO}

Objetivo: Discutir com base na literatura, os fatores associados a adesão de crianças e adolescentes com doença celíaca à terapia nutricional. Métodos: Trata-se de uma revisão da literatura, cuja busca dos artigos científicos foi realizada nas bases de dados PubMed, Scielo e Lilacs. Foram selecionados 8 artigos originais, entre os anos de 2013 a 2018, realizados em crianças e adolescentes, com diagnóstico de doença celíaca, com idades entre 02 e 20 anos, nos quais os autores abordaram sobre os diferentes fatores que influenciam a adesão à dieta livre de glúten. Resultados: A adesão estrita é fundamental para evitar o surgimento de sintomas clínicos, e obter-se sucesso no tratamento, sendo um fator variável que envolve diversos aspectos, como a idade, acompanhamento familiar e a própria escolha do paciente. A complacência alimentar a uma dieta sem glúten é mais prevalente em crianças do que em adolescentes. As transgressões de adesão envolvem o alto custo para o seguimento da dieta, principalmente em crianças, a baixa aceitação aos alimentos, falta de escolha e influência dos amigos. Considerações finais: Diante do exposto, entende-se que a adesão à dieta isenta de glúten, exige algumas mudanças alimentares, logo, enfrenta inúmeros obstáculos que devem ser vencidos.

Palavras-chave: Doença Celíaca, Terapia Nutricional, Dieta livre de glúten, Adesão.

\begin{abstract}
Objective: To discuss, based on the literature, the factors associated with children and adolescents with celiac disease adherence to nutritional therapy. Methods: This is a literature review, whose search for scientific articles was performed in the PubMed, Scielo and Lilacs databases. Were selected 08 original articles from 2013 to 2018, performed in children and adolescents, diagnosed with celiac disease, aged between 2 and 20 years, in which the authors addressed the different factors that influence adherence to gluten free diet. Results: Strict adherence is essential to prevent the appearance of clinical symptoms and to be successful in treatment, being a variable factor that involves several aspects, such as age, family support and the patient's own choice. Compliance with a gluten-free diet is more prevalent in children than in adolescents. Adherence transgressions involve the high cost of following the diet, especially in children, low acceptance of food, lack of choice and influence of friends. Final considerations: Given the above, it is understood that adherence to the gluten-free diet requires some dietary changes, thus faces numerous obstacles that must be overcome.
\end{abstract}

Keywords: Celiac Disease, Nutritional Therapy, Gluten Free Diet, Adherence.

\footnotetext{
${ }^{1}$ Universidade Federal do Piauí, Picos-PI, Parnaíba-PI e Teresina-PI. *E-mail: nutrifatimabarros@gmail.com ${ }^{2}$ Centro Universitario Uninovafapi, Teresina, Piauí.

${ }^{3}$ Faculdade de Ciências Médicas de Campina Grande, Campina Grande-PB.
} 


\section{RESUMEN}

Objetivo: Discutir, con base en la literatura, los factores asociados con la adherencia de niños y adolescentes con enfermedad celíaca a la terapia nutricional. Métodos: Esta es una revisión de la literatura, cuya búsqueda de artículos científicos se realizó en las bases de datos PubMed, Scielo y Lilacs. Se seleccionaron 8 artículos originales de 2013 a 2018, realizados en niños y adolescentes, diagnosticados con enfermedad celíaca, con edades entre 2 y 20 años, en los que los autores abordaron los diferentes factores que influyen en la adherencia a dieta sin gluten. Resultados: el cumplimiento estricto es esencial para prevenir la aparición de síntomas clínicos y tener éxito en el tratamiento, siendo un factor variable que involucra varios aspectos, como la edad, el apoyo familiar y la elección del paciente. El cumplimiento de una dieta libre de gluten es más frecuente en niños que en adolescentes. Las transgresiones de adherencia implican el alto costo de seguir la dieta, especialmente en los niños, la baja aceptación de los alimentos, la falta de elección y la influencia de los amigos. Consideraciones finales: Dado lo anterior, se entiende que la adherencia a la dieta libre de gluten requiere algunos cambios en la dieta, por lo tanto, enfrenta numerosos obstáculos que deben superarse.

Palabras clave: Enfermedad celíaca, terapia nutricional, dieta libre de gluten, adherencia.

\section{INTRODUÇÃO}

A doença celíaca (DC) consiste num distúrbio sistêmico de mecanismo imunológico, que afeta o intestino delgado de indivíduos que apresentam uma suscetibilidade genética, na qual a interação de certos fatores ambientais produz um efeito alterado da resposta imunológica (WESSELS MMS, et al., 2018). Nesses indivíduos, ocorre um processo inflamatório em resposta à ingestão do glúten (WALKER MD, et al., 2018).

As manifestações clinicas da DC clássica incluem perda de peso, desnutrição, distensão abdominal, diarreia e esteatorreia. Entretanto, a DC pode ser assintomática ou podem ocorrer manifestações sistêmicas como anemia, osteoporose, alterações do sistema neurológico e neoplasias podem ocorrer (TEIXEIRA FAM, et al., 2017). Testes sorológicos, biópsia duodenal e o alívio dos sintomas citados pós adesão a uma dieta isenta de glúten permitem a confirmação do diagnóstico de DC (WALKER MD, et al., 2018).

Antes considerada um distúrbio gastrointestinal relativamente raro que afetava quase que exclusivamente crianças brancas, a DC pode ocorrer em qualquer etnia e faixa etária, e afetar qualquer órgão do corpo (LEONARD MM, et al., 2017). Lebwohl B, et al. (2018) estimam uma prevalência global de DC de 1\% baseada principalmente em populações europeias e outras caucasianas. Nos EUA, a prevalência entre a população pediátrica é de 3-13:1000 (HASS K, et al., 2017). No Brasil, estima-se que cerca de um a cada 474 adultos e uma a cada 184 crianças apresentem a doença não diagnosticada (LIU SM, et al., 2014).

Pacientes com DC apresentam maior risco de desenvolver doenças cardíacas, ósseas, malignidades, anormalidades reprodutivas e neurológicas, e deficiências nutricionais (vitaminas B6 e B9, cálcio, zinco e ferro) (BATHRELLOU E, et al., 2018). A criança e o adolescente celíacos podem ter o crescimento e o desenvolvimento físico e mental comprometidos, já que os micronutrientes são indispensáveis ao crescimento físico, ao desenvolvimento sexual e neuromotor, e à integridade e funcionamento do sistema imunológico (PEDRAZA DF, et al., 2013).

Atualmente a única alternativa terapêutica para a DC consiste na retirada do glúten da dieta, uma família de proteínas comumente encontrada no trigo, na cevada e no centeio (TEIXEIRA FAM, et al., 2017; WALKER $M D$, et al., 2018). A dieta isenta do glúten sendo adotada de forma permanente e definitiva, melhora os sintomas e previne complicações associadas à DC na maioria dos pacientes (LIU SM, et al., 2014).

Ainda que a terapia nutricional seja o único tratamento para o celíaco, a adesão à dieta isenta de glúten é baixa, com maior risco de baixa adesão entre os adolescentes (HAAS K, et al., 2017; SCHILLING KW, et al., 2018; TEIXEIRA FAM, et al., 2017). Tanto na pediatria quanto na adolescência a dieta isenta de glúten pode ser um desafio psicológico e social afetando o bem-estar psicossocial e estilo de vida destes e de suas famílias 
(LEVRAN N, et al., 2018; RODRIGUES M, et al., 2018). Assim, o objetivo deste estudo foi discutir, com base na literatura, os fatores associados à adesão de crianças e adolescentes com doença celíaca à terapia nutricional.

\section{MÉTODOS}

Trata-se de uma revisão da literatura, cuja busca dos artigos científicos foi realizada nas bases de dados PubMed, Scielo e Lilacs. A pesquisa foi conduzida utilizando operadores lógicos para combinação dos seguintes Descritores em Ciências da Saúde (Decs).: "doença celíaca", "terapia nutricional", "adesão", "dieta livre de glúten"; bem como seus correspondentes em inglês: "celiac disease", "nutrition therapy", "adherence", "gluten-free diet".

Para elaboração desta revisão de literatura foram incluídos: artigos científicos originais que abordaram os fatores que influenciam a adesão à dieta livre de glúten por crianças e adolescentes com doença celíaca, publicados em periódicos indexados entre os anos de 2013 a 2018 e escritos nos idiomas português e inglês. Artigos de revisão, teses, dissertações, artigos originais publicados fora do período anteriormente mencionado, estudos realizados com animais e estudos realizados fora desta faixa etária foram excluídos.

A partir das palavras chaves supracitadas foram localizados 27 artigos, dos quais foram selecionados 8 com base nos critérios de inclusão e exclusão. Esta revisão constitui-se apenas de artigos científicos que tiveram a amostra composta por crianças e adolescentes com diagnóstico de doença celíaca, de ambos os sexos, com idade entre 02 e 20 anos. Considerando limitado número de estudos disponíveis que investigaram a temática apenas em crianças e adolescentes, optou-se por estender o limite de faixa etária até 20 anos completos.

\section{RESULTADOS E DISCUSSÃO}

Os artigos selecionados estão dispostos na Tabela 1, de forma a expor de forma detalhada os resultados dos mesmos. Os oito artigos trabalharam com grupos etários específicos, envolvendo crianças e adolescentes com o objetivo de investigar e avaliar aspectos inerentes à adesão de uma dieta sem glúten (DSG).

A adesão estrita à DSG é fundamental para evitar o surgimento de sintomas clínicos e, assim, obter-se sucesso no tratamento. Entretanto, a adesão à dieta é variável, podendo ser influenciada por alguns fatores tais como a faixa etária, tempo de diagnóstico da DC, percepção de desempenho no seguimento da dieta, disponibilidade, palatabilidade, custo e aspecto nutricional dos alimentos sem glúten (ALZABEN AS, et al., 2015; BRANCAGLIONI BCA, et al., 2016; RODRIGUES M, et al., 2018; SCHILLING KW, et al., 2018).

De modo geral, a adesão (ou complacência) à DSG, independentemente de qualquer fator, é referida por um número significativo de portadores de DC. Entretanto, esse número reduz quando falamos da adesão estrita, isto é, evitar todos os alimentos que tenham glúten ou resíduos de glúten em todos os momentos (ALZABEN AS, et al., 2015; CHARAMPOPOULOS D, et al., 2013; LEVRAN N, et al., 2018; RODRIGUES M, et al., 2018; SCHILLING KW, et al., 2018; WAGNER G, et al., 2016; WEBB C, et al., 2015). Como bem documentado na literatura, a melhora dos sintomas e a possibilidade de restauração da histologia do intestino delgado só ocorre quando, de fato, se evita a ingestão do glúten (WESSELS MMS, et al., 2018). Alzaben AS, et al. (2015) revelou que nem mesmo a presença de sintomas gastrointestinais estimula o celíaco para aderir ao tratamento dietético, o que dificulta ainda mais a adesão.

Apesar de ser uma doença bem descrita, a DC pode ter o diagnóstico tardio, o que pode influenciar de forma negativa a adesão dos portadores à terapia nutricional. No estudo de Rodrigues $M$, et al. (2018), 93,8\% dos indivíduos relataram sofrer de sintomas característicos da DC (principalmente diarreia) por quase 2 anos antes de serem diagnosticados. Estudo indica que quanto mais precoce ocorrer o diagnóstico, maior é a chance dos indivíduos aderirem à dieta (SCHILLING KW, et al., 2018). No estudo de Webb C, et al. (2015), a adesão autorreferida dos participantes não diferiu entre aqueles que relataram sintomas antes do diagnóstico e aqueles que não o fizeram. 
Tabela 1- Adesão e fatores associados a adesão de crianças e adolescentes com doença celíaca à terapia nutricional.

\begin{tabular}{|c|c|c|c|}
\hline AUTOR/ANO & AMOSTRA/TIPO DE ESTUDO & OBJETIVO & RESULTADOS/CONSIDERAÇŌES \\
\hline $\begin{array}{l}\text { Charalampopoulos } \\
\text { D, et al. (2013) }\end{array}$ & $\begin{array}{c}90 \text { pais de crianças e } \\
\text { adolescentes com DC } \\
(34 \mathrm{H} \text { e } 66 \mathrm{M}) \\
2-18 \text { anos } \\
\text { Estudo transversal }\end{array}$ & $\begin{array}{l}\text { Registrar as taxas de adesão e investigar } \\
\text { a conexão entre a adesão dietética e } \\
\text { dados demográficos, fatores relacionados } \\
\text { à doença e conhecimento dos pais. }\end{array}$ & $\begin{array}{l}\text { - A adesão estrita à DSG foi referida por } 44,4 \% \text { dos pais } \\
\text { das crianças e adolescentes com DC; } \\
66,7 \% \text { das crianças em idade pré-escolar (2-4 anos) } \\
\text { apresentaram adesão à DSG. Nos adolescentes essa } \\
\text { adesão representou } 34,2 \% \text {. } \\
\text { - Uma em cada duas crianças de } 5-12 \text { anos seguiu uma } \\
\text { dieta rigorosa. }\end{array}$ \\
\hline $\begin{array}{l}\text { Alzaben AS, et al. } \\
(2015)\end{array}$ & $\begin{array}{c}32 \text { indivíduos } \\
\text { com DC ( } 6 \mathrm{H} \text { e } 26 \mathrm{M}) \text { e } 32 \\
\text { controles saudáveis (13H e } \\
19 \mathrm{M}) \\
4-18 \text { anos } \\
\text { Estudo transversal }\end{array}$ & $\begin{array}{l}\text { Avaliar a adequação nutricional global da } \\
\text { DSG em crianças e adolescentes com DC } \\
\text { e comparar as ingestões com as de } \\
\text { crianças e adolescentes saudáveis. }\end{array}$ & $\begin{array}{l}\text { - } 77 \% \text { dos indivíduos com DC relataram adesão à DSG. } \\
\text { Entretanto, apenas } 23 \% \text { referiram adesão estrita à } \\
\text { DSG. } \\
\text { DSG acessível e nutricionalmente adequada foi o } \\
\text { principal desafio enfrentado pelas crianças com DC e } \\
\text { seus famíliares. }\end{array}$ \\
\hline $\begin{array}{l}\text { Webb C, et al. } \\
\text { (2015) }\end{array}$ & $\begin{array}{c}210 \text { indivíduos } \\
(90 \mathrm{H} \text { e } 120 \mathrm{M}) \\
12 \text { anos } \\
\text { Subestudo de estudo } \\
\text { transversal de rastreamento }\end{array}$ & $\begin{array}{l}\text { Avaliar a mudança nos níveis de TG2-IgA } \\
\text { e a adesão a DSG após um ano de } \\
\text { seguimento em crianças com DC } \\
\text { detectada por triagem na população geral. }\end{array}$ & $\begin{array}{l}\text { - Das } 193 \text { crianças que completaram o questionário de } \\
\text { adesão, } 82 \% \text { relataram adesão à DSG, sendo que isso } \\
\text { foi semelhante entre os sexos; } \\
\text { - No seguimento, } 75 \% \text { dos adolescentes relatou adesão } \\
\text { à DSG e apresentaram níveis normais de TG2-lgA } \\
\text { (<5U/mL). No entanto, } 6,7 \% \text { dos adolescentes que } \\
\text { relataram adesão à DSG, não apresentaram TG2-lgA } \\
\text { normalizado após um ano (TG2-lgA de } 5,2 \text { a } \\
10,9 U / m L) \text {. } \\
2 \text { adolescentes relataram não seguir uma DSG, porém } \\
\text { apresentaram níveis normais de TG2-lgA (<5 } \mathrm{U} / \mathrm{mL} \text { ). }\end{array}$ \\
\hline $\begin{array}{l}\text { Brancaglioni BCA, } \\
\text { et al. (2016) }\end{array}$ & $\begin{array}{c}5 \text { indivíduos } \\
(2 \mathrm{H} \text { e } 3 \mathrm{M}) \\
10 \text { a } 16 \text { anos } \\
\text { Estudo qualitativo, exploratório } \\
\text { e descritivo }\end{array}$ & $\begin{array}{l}\text { Compreender a experiência de crianças e } \\
\text { adolescentes que convivem com DM1 e } \\
\text { DC. }\end{array}$ & $\begin{array}{l}\text { - Entre as crianças a adesão à DSG é dificultada pela } \\
\text { restrição das escolhas alimentares e pelo alto custo, } \\
\text { especialmente naquelas de famílias com menor poder } \\
\text { aquisitivo; } \\
\text { - Entre os adolescentes, o constrangimento e as } \\
\text { dificuldades no convívio social influenciam a adesão à } \\
\text { DSG. }\end{array}$ \\
\hline
\end{tabular}




\begin{tabular}{|c|c|c|c|}
\hline $\begin{array}{c}\text { Wagner G, et al. } \\
(2016)\end{array}$ & $\begin{array}{c}281 \text { indivíduos } \\
\text { com DC (73H e 208M) e } 95 \\
\text { controles saudáveis } \\
(32 \mathrm{H} \text { e } 63 \mathrm{M}) \\
10 \text { a } 20 \text { anos } \\
\text { Estudo transversal }\end{array}$ & $\begin{array}{l}\text { Avaliar as estratégias de enfrentamento } \\
\text { aplicadas em situações específicas da } \\
\text { doença e dimensões de personalidade } \\
\text { em adolescentes com DC comprovada } \\
\text { por biópsia e comparar Pacientes } \\
\text { Aderentes (PA) e Pacientes não } \\
\text { aderentes (PNA) à DSG. }\end{array}$ & $\begin{array}{l}\text { - } 80,8 \% \text { dos adolescentes relataram adesão à DSG; } \\
\text { - } 19 \% \text { dos adolescentes não aderiram estritamente à } \\
\text { DSG; } \\
\text { - Os PA à DSG apresentaram menor uso de estratégias } \\
\text { de enfrentamento emocional, apresentando menor } \\
\text { impulsividade e maior perfeccionismo do que os PNA. }\end{array}$ \\
\hline $\begin{array}{c}\text { Levran N, et al. } \\
(2018)\end{array}$ & $\begin{array}{c}40 \text { indivíduos } \\
\text { com DC }(19 \mathrm{H} \text { e } 21 \mathrm{M}) \text { e } 15 \\
\text { controles saudáveis }(9 \mathrm{H} \text { e } 6 \mathrm{M}) \\
4-15 \text { anos } \\
\text { Estudo prospectivo de } \\
\text { intervenção clínica }\end{array}$ & $\begin{array}{l}\text { Avaliar a influência da DSG sobre os } \\
\text { hábitos alimentares e comportamentos de } \\
\text { estilo de vida da criança e de sua família. }\end{array}$ & $\begin{array}{l}\text { - } 70 \% \text { dos pais relataram que filhos nunca comeram } \\
\text { glúten conscientemente; } \\
\text { - } 50 \% \text { dos pais referiram que os filhos comem alimentos } \\
\text { que possuem resíduos de glúten. }\end{array}$ \\
\hline $\begin{array}{l}\text { Rodrigues M, et al. } \\
\text { (2018) }\end{array}$ & $\begin{array}{l}35 \text { indivíduos } \\
(11 \mathrm{H} \mathrm{e} 24 \mathrm{M}) \\
<20 \text { anos } \\
\text { Estudo transversal e } \\
\text { retrospectivo }\end{array}$ & $\begin{array}{l}\text { Examinar a taxa e os determinantes da } \\
\text { não adesão a uma DSG, bem como os } \\
\text { hábitos alimentares e o estado nutricional } \\
\text { de crianças e adolescentes } \\
\text { diagnosticados com DC em um centro de } \\
\text { referência terciário. }\end{array}$ & $\begin{array}{l}\text { - Todos os indivíduos relataram ser instruídos a } \\
\text { aderirem à DSG, mas } 20 \% \text { relataram não evitar o } \\
\text { glúten em todos os momentos; } \\
\text { - } 53,8 \% \text { dos participantes relataram que a não adesão } \\
\text { à DSG se deve a escolha voluntária. Entretanto, } \\
\text { 30,8\% referiram que o alto custo e a baixa aceitação } \\
\text { da DSG são determinantes da não adesão; } \\
\text { - } 51,9 \% \text { relataram que as transgressões à dieta foram } \\
\text { intencionais. }\end{array}$ \\
\hline $\begin{array}{c}\text { Schilling KW, et al. } \\
\text { (2018) }\end{array}$ & $\begin{array}{l}65 \text { indivíduos } \\
\quad(25 \mathrm{H} \mathrm{e} 40 \mathrm{M}) \\
<18 \text { anos } \\
\text { Estudo transversal e analítico }\end{array}$ & $\begin{array}{l}\text { Avaliar os fatores que influenciam a } \\
\text { adesão à DSG em pacientes pediátricos e } \\
\text { medir a concordância entre os resultados } \\
\text { sorológicos e um questionário de adesão } \\
\text { nutricional. }\end{array}$ & $\begin{array}{l}\text { - } 52,3 \% \text { dos entrevistados relataram adesão à DSG. } \\
\text { Porém, a sorologia indicou a adesão de apenas } 44 \% \text {, } \\
\text { e conforme o questionário de adesão somente } 30,8 \% \\
\text { aderiram estritamente à DSG; } \\
\text { - Crianças com diagnóstico de DC antes dos } 2 \text { anos de } \\
\text { idade apresentaram maior adesão à DSG }(60,7 \%) \text { do } \\
\text { que as diagnosticadas em idades posteriores. } \\
\text { - A percepção de mau desempenho na DSG aumentou } \\
\text { em } 3,4 \text { o risco dos entrevistados não aderirem à DSG } \\
\text { do que a percepção de bom desempenho. } \\
\text { - A participação em eventos sociais não aumentou o } \\
\text { risco de transgressões da DSG. }\end{array}$ \\
\hline
\end{tabular}


Seguir uma dieta isenta de alimentos fonte de glúten parece ser complexo em todas as faixas etárias, porém na adolescência pode ser mais difícil ao considerarmos as pressões sociais, culturais, econômicas e práticas (HOMMEL KA, et al., 2008). Contudo, no estudo realizado por Rodrigues M, et al. (2018), 45,7\% dos adolescentes referiu que o nível de dificuldade para aderir a uma DSG é pequeno e/ou médio, e 37\% destes relatou que não há dificuldade na adesão, sugerindo que não aderir à DSG pode ser algo voluntário entre os adolescentes. No entanto, dificuldades impostas pela DSG no cotidiano e convívio dos adolescentes com os amigos, como o sentimento de vergonha ao frequentaram ambientes em que sua patologia é desconhecida, também parece contribuir negativamente para a não aderência à dieta nesse público (BRANCAGLIONI BCA, et al., 2016).

Nesse contexto, um estudo realizado por Hass K, et al. (2017) avaliou o impacto de uma intervenção em adolescentes com DC, onde mensagens de texto foram utilizadas para impulsionar e/ou melhorar a adesão dos participantes ao tratamento dietético. Os autores observaram que essa estratégia, apesar de não influenciar significativamente na adesão, demonstrou-se eficaz para melhorar a qualidade de vida por meio do compartilhamento de informações acerca do tratamento.

Indivíduos com menor idade parecem aderir mais a DSG (CHARAMPOPOULOS D, et al., 2013). Isso pode representar uma relação positiva e fortalecedora do conhecimento e da participação dos pais no tratamento dietético de crianças com DC. No entanto, vale destacar que nesse estudo, a maioria dos questionários foram respondidos pelas mães das crianças, o que pode ou não ter interferência nas taxas de adesão, já que, coincidentemente, as mães das crianças que foram aderentes a DSG tinham mais conhecimento sobre a doença. Brancaglioni BCA, et al. (2016) destaca que o alto custo e a restrição de escolhas alimentares de uma dieta isenta de glúten são fatores de risco para que as crianças cometam transgressões a terapia nutricional.

A ingestão de alimentos sem glúten está associada à baixa ingestão de fibras, folato, vitamina $D$ e vitamina $\mathrm{K}$ e à alta ingestão de proteína, açúcar e gordura saturada (KAUTTO E, et al., 2014). No estudo de Alzaben AS, et al. (2015) que avaliou a qualidade nutricional da DSG em crianças e adolescentes celíacos, aqueles aderentes à DSG tiveram maior ingestão de energia, carboidratos de alto indice e carga glicêmica, fibras, vitamina $\mathrm{C}, \mathrm{B} 6$ e ferro e menor consumo de folato do que os controles saudáveis.

Levran N, et al. (2018) também demonstrou que a adesão à DSG pode promover mudanças negativas do ponto de vista nutricional nos hábitos alimentares dos celíacos, propiciando um ambiente mais obesogênico. Nesse estudo, o consumo de lanches entre as refeições, doces, bolos e sorvetes aumentou na maioria das famílias dos participantes.

As modificações da dieta supracitadas podem contribuir positivamente com o ganho de peso, como demonstrado por Levran N, et al. (2018), onde a proporção de pacientes com DC com sobrepeso e obesidade aumentou de 7,5\% para 12,5\% após a DSG. De modo semelhante, Rodrigues M, et al. (2018) relatou aumento no número de crianças com sobrepeso ou obesidade ao longo do seguimento desses pacientes. Entretanto, os autores ressalvam que não houve diferença nos parâmetros nutricionais (IMC/idade e estatura/idade) entre os pacientes que relataram transgressão da dieta e os que demonstraram adesão ao tratamento. Vale destacar que no referido estudo, a amostra foi pequena e os dados sobre a evolução nutricional dos participantes foram incompletos, o que segundo os próprios autores, pode ter interferido na ausência de associação entre transgressão e outras variáveis, incluindo o estado nutricional.

Como já bem descrito, a principal dificuldade em conviver com a DC está na adesão a DSG. Neste contexto, um estudo de Rajpoot $P$, et al. (2015) avaliou a adesão ao tratamento para portadores de doença celíaca com antes e após estratégias de aconselhamento. Em pacientes que já faziam uso da dieta sem glúten, puderam perceber que com 6 meses de aconselhamento, o nível de adesão "bom" ou "excelente", aumentou de $53,3 \%$ para $92,9 \%$. E, para os pacientes que ainda não tinham iniciado o tratamento, o resultado pós-aconselhamento registrou $64,8 \%$ de adesão após o primeiro mês de aconselhamento e, após 6 meses esse percentual aumentou para $96,3 \%$. Assim, o aconselhamento pode ser uma ferramenta importante para contribuir para melhores níveis de adesão ao tratamento para celíacos. 


\section{CONSIDERAÇÕES FINAIS}

A adesão à dieta isenta de glúten, exige algumas mudanças alimentares, logo, enfrenta alguns obstáculos que devem ser vencidos. As crianças apresentam menor resistência ao tratamento, e, isso pode ter relação com a influência do conhecimento dos pais acerca da doença. Em adolescentes a resistência à dieta é maior e, isso pode envolver fatores sociais e afetivos, e, resultar numa escolha voluntária de não aderir à dieta. Diante disso, entende-se que a participação dos pais pode exercer influência positiva sobre a adesão e sucesso do tratamento. E, para os adolescentes, é necessário que se desenvolvam estratégias eficazes de conscientização da importância do tratamento para assim, facilitar a adesão e continuidade do tratamento.

\section{REFERÊNCIAS}

1. ALZABEN AS, et al. Assessing nutritional quality and adherence to the gluten-free diet in children and adolescents with celiac disease. Canadian Journal of Dietetic Practice and Research, 2015; 76(2): 5663.

2. BATHRELLOU E, et al. Celiac disease and non-celiac gluten or wheat sensitivity and health in later life: A review. Maturitas, 2018; 112: 29-33.

3. BRANCAGLIONI BCA, et al. Crianças e adolescentes que convivem com diabetes e doença celíaca. Revista Gaúcha Enfermagem, 2016; 37(1): e53787.

4. CHARALAMPOPOULOS D, et al. Determinants of adherence to gluten-free diet in Greek children with coeliac disease: a cross-sectional study. European journal of clinical nutrition, 2013; 67(6): 615.

5. HAAS K, et al. Text message intervention (teach) improves quality of life and patient activation in celiac disease: a randomized clinical trial. J Pediatric, 2017; 185: 62-67.

6. HOMMEL KA, et al. Treatment regimen adherence in pediatric gastroenterology. J Pediatr Gastroenterol Nutr., 2008; 47(5): 526-43.

7. KAUTTO E, et al. Nutrient intake in adolescent girls and boys diagnosed with coeliac disease at an early age is mostly comparable to their non-coeliac contemporaries. J Hum Nutr Diet., 2014; 27(1): 41-53.

8. LEBWOHL B, et al. Coeliac disease. Lancet, 2018; 391(10115): 70-81.

9. LEONARD MM, et al. Celiac disease and nonceliac gluten sensitivity: a review. JAMA, 2017; 318(7): 647-656.

10. LEVRAN N, et al. Obesogenic habits among children and their families in response to initiation of gluten-free diet. Eur J Pediatr., 2018; 177(6): 859-866.

11. LIU SM, et al. Doença celíaca. Rev Med Minas Gerais, 2014; 24(Supl 2): S38-S45.

12. PEDRAZZA DF, et al. Crescimento e deficiências de micronutrientes: perfil das crianças assistidas no núcleo de creches do governo da Paraíba, Brasil. Ciência \& Saúde Coletiva, 2013; 18(11): 3379-3390.

13. RAJPOOT $P$, et al. Adherence to gluten-free diet and barriers to adherence in patients with celiac disease. Indian Journal of Gastroenterology, Springer Nature, 2015; 34(5): 380-386.

14. RODRIGUES M, et al. Rate and determinants of non-adherence to a gluten-free diet and nutritional status assessment in children and adolescentes with celiac disease in a tertiary Brazilian referral center: a cross-sectional and retrospective study. BMC Gastroenterology, 2018; 18:15.

15. SCHILLING KW, et al. Perception of following gluten-free diet and adherence to treatment in pediatric patients with celiac disease. Rev Chil Pediatr., 2018; 89(2): 216-223.

16. TEIXEIRA FAM, et al. Dieta isenta de glúten e risco de desfechos gestacionais desfavoráveis em mulheres com doença celíaca: revisão sistemática. Reprod Clim., 2017; 32(2): 120-126.

17. WAGNER G, et al. Coeliac disease in adolescence: Coping strategies and personality factors affecting compliance with gluten-free diet. Appetite, 2016; 101: 55-61.

18. WALKER MD, et al. Endocrine complications of celiac disease: a case report and review of the literature. Endocr Res., 2018; 10: 1-19.

19. WEBB $C$, et al. High adherence to a gluten-free diet in adolescents with screening-detected celiac disease. Journal of pediatric gastroenterology and nutrition, 2015; 60(1): 54-59.

20. WESSELS MMS, et al. Assessment of dietary compliance in celiac children using a standardized dietary interview. Clin. Nutr., 2018; 37(3): 1000-1004. 